\title{
Modelo de regresión logística aplicado a un estudio sobre enfermedad de Chagas
}

\author{
A logistic regression model applied \\ to Chagas' disease
}

Gabriela Boggio 1

\footnotetext{
1 Instituto de Investigaciones Teóricas y Aplicadas en Estadística, Facultad de Ciencias Económicas y Estadística, Universidad Nacional de Rosario. Bv. Oroño 1261, C.P. 2000, Rosario, Argentina.
}

Abstract The focus of this paper is the application of statistical models to the study of socioeconomic conditioning factors in perinatal Chagas' di sease conducted in Rosario, Argentina. A case (154) and control (158) design was applied to investigate socioeconomic and cultural differences in pregnant women in Hospital Roque Sáenz Peña as to their infection status. Logistic regression model s were used to eval uate the importance of antecedents linked to the infection and socioeconomic and cultural factors for infection status. For pregnant women, the importance of antecedents linked to the infection was confirmed and the women's level of schooling stood out as the predominant socioeconomic condition associated with infection. Log-linear models were used to explore the associations between certain explanatory variables. This approach pointed up the most rel evant associations between such factors and Chagas' disease and provided a better understanding of the framework of relationships among them.

Key words Case-Control Studies; Logistic Regresion; Log-Linear Models; Chagas' Disease

Resumen El propósito de esta presentación es mostrar la aplicación de modelos estadísticos en un estudio sobre las implicancias de los condicionantes socioeconómi cos en el Chagas connatal realizado en Rosario. A fin de indagar diferencias socioeconómi cas y culturales en embarazadas del Hospital Roque Sáenz Peña según la condición de infectada o no infectada, se adoptó un diseño de casos (154) y controles (158). Se utilizaron model os de regresión logística con la intención de valorar la importancia de los atributos referidos a los antecedentes ligados a la infección y de los factores socioeconómi cos y culturales en relación con la condición de infección. Esta modelización permitió corrobar, para esta población de mujeres, la importancia de los antecedentes ligados a la infección; se destacó el nivel de instrucción de la mujer como condicionante soci oecónomico preponderante vinculado con la infección. Para ahondar en las asociaciones entre ciertas variables explicativas, se completó el análisis con model izaciones logl ineales. El recurso analítico empleado permitió no sól o hallar las asociaciones más rel evantes entre los factores consi derados y la enfermedad de Chagas, sino también lograr una mejor comprensión acerca de la trama de relaciones existentes entre ellos.

Palabras clave Estudio de Casos y Controles; Regresión Logística; Modelos Logl ineales; Enfermedad deChagas 


\section{Introducción}

La enfermedad de Chagas, originalmente típica de las áreas rurales, ha pasado a ser un problema que también atañe a las grandes ciudades fundamentalmente debido a la migración rural-urbana y a sus consecuencias sociales.

En Argentina esta enfermedad es endémica en gran parte del territorio, varía su prevalencia según la zona geográfica, pero la información disponible refiere a grupos específicos y no a la población en general. Las estimaciones del total de nuevos casos a nivel nacional se basan en proyecciones a partir de grupos poblacionales donde las mujeres tienen escasa participación : ingresantes al servicio militar, exclusivo para varones hasta el año 1994; dadores de sangre, donde se ha verificado la débil presencia de las mujeres como consecuencia de cuestiones culturales (Torres de Quinteros et al., 1990) y niños menores de 4 años en ocasión de campañas de prevención.

Así es que prácticamente la única oportunidad de diagnóstico de la enfermedad que tiene la mujer se concreta ante un embarazo, cuando realiza los controles prenatales y en la medida en que se incluya el test de Chagas en la rutina de dicho control. Las cifras disponibles sobre la prevalencia de la infección en embarazadas varía de un 3 a un 30\%, de acuerdo al área estudiada (Barousse et al., 1978).

En lo que refiere al riesgo de transmisión connatal, las investigaciones realizadas en Argentina muestran resultados variables según las regiones, alcanzando niveles del $4 \%$ en áreas endémicas (Zaidenberg \& Segovia, 1993).

El Chagas congénito aparece como problema en estudios de carácter básicamente clínico-biológico y que, en su mayor parte, se restringen a un número reducido de casos (Azogue et al., 1985; Barousse et al., 1978; Bittencourt, 1976; Lorca et al., 1987; Moya et al., 1985; Saleme et al., 1971; Zai denber \& Segovia, 1993).

Existen algunos estudios, en especial en Brasil, que abordan la problemática del Chagas mediante la evaluación de factores de riesgo tanto socioeconómicos, familiares, como medio-ambientales. La consideración simultánea de esta multiplicidad de factores ha implicado la selección de metodología estadística del tipo de los modelos loglineales, en algunos casos (Starr et al., 1991), la regresión logística ya sea condicional o no condicional, según el diseño de la investigación, en otros estudios (Andrade et al., 1995; Martelli et al., 1992), o bien la elección de alguna modelización que permitiera considerar supuestos causales teóricos tales como los sistemas recursivos de model os logit (Torres de Quinteros et al., 1990).

En esta línea se inscribe la investigación realizada en Rosario por el Centro de Estudios Sanitarios y Sociales (Troncoso et al, 1995) que, con el propósito de indagar diferencias socioeconómicas y culturales en embarazadas según su condición de infectadas o no infectadas, utiliza model os de regresión logística y, en forma complementaria, modelos loglineales.

El objetivo mencionado forma parte de un proyecto más amplio que pretendió esclarecer el problema del Chagas connatal en un área urbana, sin transmisión vectorial demostrada, pero receptora de migrantes provenientes de zonas endémicas, para la elaboración de estrategias de intervención apropiadas.

\section{Consideraciones sobre el diseño de la investigación}

El estudio se realizó en el Hospital Municipal Roque Sáenz Peña, ubicado en la zona sudeste de la ciudad de Rosario. El área de influencia del Hospital es amplia y asiste la demanda espontánea de la población y los casos derivados de los Centros de Salud, en carácter de primer eslabón de referencia. También asiste la demanda de la población de localidades vecinas a la ciudad, fundamentalmente Villa Gobernador Gálvez.

El universo quedó definido, a los fines de la implementación del estudio, por toda mujer embarazada que acudió al Hospital en el período febrero 1993 - enero 1994. A lo largo del período de captación se practicaron 3.166 estudios serológicos, siendo la tasa de infección encontrada igual al 5,2\%. El período definido fue elegido de acuerdo a la cantidad de partos ocurridos y a las tasas de infección conocidas de manera de poder obtener una casuística mínima indispensable a los fines analíticos. Se intentó de esta manera conciliar costo-tiempo y factibilidad analítica (Andrade et al., 1994).

Para identificar aquellos aspectos o situaciones más estrechamente ligados a la enfermedad de Chagas, se construyó un diseño de casos y controles de carácter retrospectivo. Este diseño se consideró como el más apropiado ya que un grupo control es absolutamente esencial para evitar asociaciones erróneas que lleven a atribuir ciertas características como atributos de las madres infectadas, cuando en realidad dichos atributos se hallan también presentes, y en la misma medida, en las no infectadas. Se descartó un diseño de grupos apareados, porque la evaluación empírica de los 
diferentes factores que influyen en ambos grupos es un resultado de interés.

El grupo de casos quedó constituido por 154 mujeres diagnosticadas como seropositivas en el período definido ( 9 mujeres seropositivas no pudieron incorporarse al estudio o porque optaron por otras instituciones en el momento del parto o por deficiencias en el registro de domicilios). El grupo control, en tanto, se construyó a partir de una selección aleatoria sistemática de las mujeres seronegativas. Esta selección se realizó en forma secuencial a medida que las mujeres infectadas eran detectadas por laboratorio, es decir, paralelamente a la constitución del grupo de casos., e incluyó un total de 158 mujeres seronegativas.

El criterio utilizado para arribar a un diagnóstico serológico positivo en las embarazadas se basó en dos pruebas de screening: Hemaglutinación Indirecta a título 1/ 32 y Látex. Cuando al menos una de estas dos pruebas resultaba positiva, se realizaba la confirmación a través del Test de Elisa. Cabe mencionar que el diagnóstico serológico de Chagas quedó incorporado a la rutina solicitada a toda embarazada en su primer contacto con el Hospital a partir de la ejecución de esta investigación.

La información fue recolectada a través de diversos procedimientos. Se administró un cuestionario a casos y controles y se relevó información de fuente secundaria, historias clínicas y registros del hospital. El conjunto de variables que se contemplaron fue muy amplio, abarcando tanto aspectos socioeconómicos y culturales, cuidados y creencias de la mujer acerca del embarazo, su salud y la de su hijo y la enfermedad de Chagas, como antecedentes vinculados a la infección, antecedentes obstétricos y condiciones biológicas del niño al nacer.

Esta presentación se restringe al análisis de las relaciones entre la condición de infección y un conjunto limitado de variables correspondientes a aspectos socioeconómicos y demográficos y a antecedentes vinculados a la infección, material recabado de la encuesta realizada.

\section{Metodología}

En este estudio el requerimiento de modelizar una variable respuesta del tipo mujer infectada o no infectada en función de un conjunto numeroso de variables explicativas ante un diseño epidemiológico específico, el de casos y controles, se satisface mediante el ajuste de modelos de regresión logística, en concordan- cia con otros trabajos referidos a la infección chagásica (Andrade et al., 1995; Martelli et al., 1992).

La regresión logística es el método de análisis adecuado cuando se necesita modelizar una variable respuesta binaria, del tipo presencia o ausencia de enfermedad, y permite el uso conjunto de covariables de tipo categórico y continuo, proporcionando interpretación biológica a sus parámetros (Hosmer \& Lemeshow, 1989).

En ella se suele simbolizar con $Y$ a la variable respuesta, del tipo presencia $(Y=1)$ o ausencia $(Y=0)$ de enfermedad y con $\pi(X)$ a la siguiente probabilidad: $P(Y=1 / X)$, donde $X$ es un vector de $p$ covariables.

El modelo de regresión logística múltiple está dado por: $g(X)=\beta_{0}+\beta_{1} X_{1}+\ldots . .+\beta_{p} X_{p}$ donde: $g(X)=\ln \frac{\pi(X)}{1-\pi(X)}$

En el proceso de selección del modelo se tienen en cuenta los siguientes aspectos: a) análisis univariado de las variables; b) selección de escala apropiada para las variables continuas, de modo de elegir la expresión funcional más lógica para el modelo; y c) consideración de términos de interacción, basada tanto en criterios estadísticos como teóricos. Respecto de la prueba de bondad de ajuste, se elige en este estudio la de Hosmer-Lemeshow. Asimismo se categorizan las variables continuas involucradas ajustando el modelo "logit" resultante, como forma alternativa de corroborar el "test" de bondad de ajuste.

Cuando se desean analizar las interacciones entre las variables en estudio, siendo ellas categóricas, esto es, no se pretende distinguir entre respuesta y explicativas, se utilizan los modelos loglineales.

El modelo loglineal completo en el caso tridimensional IXJXK es el siguiente:

In $\mathrm{m}_{\mathrm{ijk}}=\mathrm{u}+\mathrm{u}_{1(\mathrm{i})}+\mathrm{u}_{2(\mathrm{j})}+\mathrm{u}_{3(\mathrm{k})}+\mathrm{u}_{12(\mathrm{ij})}+\mathrm{u}_{13(\mathrm{ik})}+$ $\mathrm{u}_{23(\mathrm{jk})}+\mathrm{u}_{123(\mathrm{ijk})}$

donde $m_{i j k}$ es la frecuencia esperada para la celda $(i, j, k)$ y los u-es son los efectos principales e interacciones entre las variables.

La bondad del ajuste del model o se realiza con la estadística de razón de verosimilitud (Agresti, 1990).

Luego, para comprender mejor la interrelación entre algunas variables explicativas, se opta por los modelos loglineales, pero no tratados de manera disociada a la regresión logística; por el contrario, interesa potencializar estas técnicas de modelización en torno a un problema tan complejo como el del Chagas.

Tanto los parámetros de los modelos loglineales como los de la regresión logística están 
directamente relacionados con la medida de asociación denominada razón de "odds" (RO). La importancia de la misma en los estudios epidemiológicos radica en que, cuando la probabilidad del resultado de interés - presencia de enfermedad - es muy pequeña, esta medida es una buena estimación del riesgo relativo, tan frecuentemente empleado en este campo al permitir valorar factores de riesgo.

Por otro lado, en los estudios retrospectivos, primeramente se identifican los casos, luego se eligen los controles, y finalmente se los compara para indagar cómo difieren. De este modo, los totales para la variable respuesta - presencia o ausencia de enfermedad - están fijos en lugar de estarlo los totales correspondientes a una o más variables explicativas. Aún así, se pueden estimar los efectos de las variables explicativas sobre la variable respuesta a partir de propiedades asociadas con la RO. Esto es, si se considera el caso de una tabla $2 \times 2$ y se denomina con D y Dc a la presencia y ausencia de la enfermedad, respectivamente, y con A y Ac, a la presencia o ausencia de al guna característica posible causa, en un estudio prospectivo, se fijan las proporciones asociadas con A y Ac y luego se observan las correspondientes $D$ y Dc.

- La RO entre las dos variables es:

$\mathrm{RO}_{\text {pro }}=\mathrm{P}(\mathrm{D} / \mathrm{A}) \mathrm{P}(\mathrm{Dc} / \mathrm{Ac})$

$$
P(D c / A) P(D / A c)
$$

En el estudio retrospectivo, fijadas las proporciones para D y DC, la RO para esta situación es:

$$
\mathrm{RO}_{\text {ret }}=\frac{P(A / D) P(A c / D c)}{P(A c / D) P(A / D c)}
$$

Invocando el Teorema de Bayes, surge que $\mathrm{RO}_{\text {pro }}=\mathrm{RO}_{\text {ret }}$, y así el estudio retrospectivo mide la relación entre las variables explicativa y respuesta del mismo modo que el estudio prospectivo (Fienberg, 1981).

A partir de esta propiedad de invariancia de la RO, se demuestra que el análisis de los datos provenientes de un estudio retrospectivo vía regresión logística se realiza de la misma manera que en los estudios prospectivos (Farewell, 1979).

Para el tratamiento computacional de los datos se empleó el programa SPSS-PC+(Norusis, 1986) y para la modelización estadística específica, los programas LOGLIN (Olivier \& Neff, 1976) y MULTLR (Campos \& Franco, 1989).

\section{Resultados}

\section{Estudio preliminar}

La caracterización social de las mujeres que en general acuden al hospital corresponde al perfil típico de la población de escasos recursos, con necesidades básicas insatisfechas, como consecuencia de su precaria inserción en el mercado laboral.

Sin embargo, la comparación, uno a uno, de los distintos aspectos socioeconómicos indagados para casos y controles permite comprobar que esta situación crítica se acentúa cuando se trata de mujeres infectadas, en forma manifiesta en algunos casos, y sólo incipientemente en otros.

En el grupo de casos se registra una mayor presencia de mujeres con escasa o ninguna instrucción, grupos familiares muy extensos con probable mayor carga económica para el o los responsables del grupo familiar, sin cobertura de riesgos de salud, que permiten verificar a veces diferencias estadísticamente significativas entre ambos grupos, como se ilustra en la tabla 1. El mayor grado de criticidad de los casos se aprecia más claramente cuando se comparan sus condiciones materiales de vida, con mayores porcentajes de mujeres residentes en villas de emergencia, sin provisión de agua o sin baño.

Por último puede mencionarse que, tanto entre los casos como entre los controles, la mayoría de las mujeres no trabajan o lo hacen irregularmente y con baja carga horaria. Consecuentemente, en ambos grupos fueron escasas las mujeres que se identificaron como principal responsable del sustento económico familiar (7 seropositivas y 8 controles), reconociendo prácticamente todas las demás como tal a su pareja $(83,8 \%$ de las positivas y $73, .4 \%$ de las negativas).

En cuanto a las características de la actividad laboral del principal responsable, se observa un comportamiento similar en ambos grupos, siendo la forma de inserción más generalizada la de asalariado precario.

La contrastación de los resultados relativos a los antecedentes vinculados a la infección para casos y controles permite confirmar, por una parte, hallazgos obtenidos previamente (Torres de Quinteros et al., 1990; 1992) en lo que refiere a la existencia de una asociación positiva entre la enfermedad de Chagas y la condición de migrante, y, en particular, de migrante proveniente de zonas endémicas (77,9\% de los casos son migrantes $-66,0 \%$ de zonas endémicas - vs. $41,8 \%$ - 31,0\% de zonas endé- 
Tabla 1

Condiciones demográficas y socioeconómicas más desfavorables para casos y controles.

\begin{tabular}{|c|c|c|c|}
\hline & $\begin{array}{c}\text { Casos }(n=154) \\
\%\end{array}$ & $\begin{array}{c}\text { Controles }(n=158) \\
\%\end{array}$ & Probabilidad \\
\hline \multicolumn{4}{|l|}{ Edad } \\
\hline menor de 20 años & 14,3 & 29,1 & $0,00^{a}$ \\
\hline \multicolumn{4}{|l|}{ Grupo familiar } \\
\hline familias con 7 ó más miembros & 26,0 & 22,8 & $0,28^{b}$ \\
\hline sin pareja & 14,3 & 22,2 & $0,07^{b}$ \\
\hline sin cobertura de salud & 94,2 & 90,5 & $0,23^{b}$ \\
\hline \multicolumn{4}{|l|}{ Nivel de instrucción } \\
\hline primaria, incompleta o analfabeta & 56,5 & 29,8 & $0,00^{a}$ \\
\hline \multicolumn{4}{|l|}{ Vivienda } \\
\hline en villas de emergencia & 21,4 & 13,9 & $0,00^{b}$ \\
\hline sin provisión de agua & 22,1 & 8,9 & $0,00^{b}$ \\
\hline sin baño & 7,8 & 3,8 & $0,13^{b}$ \\
\hline
\end{tabular}

a Asociada al test de Wilcoxon.

b Asociada al test Chi cuadrado.

Tabla 2

Antecedentes ligados a la infección para casos y controles.

\begin{tabular}{|c|c|c|c|c|}
\hline Antecedente & $\begin{array}{c}\text { Casos }(n=154) \\
\%\end{array}$ & $\begin{array}{c}\text { Controles }(n=158) \\
\%\end{array}$ & Razón de “odds" & Probabilidad ${ }^{a}$ \\
\hline Migrante & 77,9 & 41,8 & 2,5 & 0,0000 \\
\hline Nacimiento en área endémica ${ }^{b}$ & 61,2 & 27,9 & 4,1 & 0,0000 \\
\hline Residencia en área endémica c & 66,0 & 31,0 & 4,4 & 0,0000 \\
\hline Transfusiones de sangre ${ }^{d}$ & 22,9 & 8,2 & 3,3 & 0,0004 \\
\hline Familiares consanguíneos con Chagas ${ }^{e}$ & 35,2 & 6,3 & 8,1 & 0,0000 \\
\hline
\end{tabular}

a Asociadas al test Chi-cuadrado.

b Se eliminan 2 casos para los que se ignora la endemicidad del lugar.

c Se elimina 1 caso para el que se ignora la endemicidad del lugar.

d Se elimina 1 caso para el que se desconoce si recibió o no transfusiones.

e Se eliminan 26 casos y 14 controles para los que se desconocía si tenían familiares chagásicos.

micas). Asimismo se verifican diferencias significativas entre casos y controles al considerar el antecedente de familiares consanguíneos con Chagas (35,2\% vs. 6,3\%) o víctimas de muerte súbita (20,6\% vs. 5,4\%).

Por otra parte, se comprueba aquí la asociación entre el antecedente de transfusiones de sangre y la presencia de la infección, no verificada anteriormente en Rosario (22,9\% vs. $8,2 \%)$. Muy probablemente la posibilidad de verificar esta diferencia se relaciona con la definición de población objetivo adoptada para este estudio. En tanto en el primer estudio la indagación se basó en resultados sobre población abierta (adultos mayores de 15 años residentes en zonas de villas de emergencia), ahora la delimitación a mujeres en edad reproductiva, con mayor riesgo de transfusión en oportunidad de partos previos, favoreció muy posiblemente la reunión de una mayor casuística (Tabla 2).

\section{Modelización estadística}

Con el propósito de valorar la importancia de los atributos referidos a los antecedentes ligados a la infección y de los condicionantes socioeconómicos y culturales en relación con la condición de infección, se realizó una serie de análisis multivariados. 
Se comenzó con la construcción de un modelo de regresión logística. El análisis realizado en el estudio preliminar, que relaciona individualmente cada factor con la condición de infección, sirvió de primer tamiz para la selección de las variables a incluir en el modelo. Así es que las variables consideradas fueron: condición de infección, variable respuesta (con categorías no infectada e infectada); residencia en villas de emergencia (con categorías no y si); disponibilidad de agua (con categorías si dispone y no dispone); nivel de instrucción (con categorías secundaria incompleta o más, primaria completa y primaria incompleta o no asistió a la escuela); transfusiones de sangre, variable discreta; edad de la mujer, variable continua y, por último, años de residencia en áreas endémicas, variable continua.

De acuerdo al análisis realizado para la selección de la escala más adecuada de cada variable, se tomó la variable transfusiones de sangre como dicotómica según la mujer contara o no con ese antecedente, en función de la escasa frecuencia de mujeres con más de una transfusión. En cuanto a los años de residencia en áreas endémicas, su categorización y posterior análisis gráfico sugirió que la mejor representación estaba dada por un polinomio de segundo grado. Con respecto a la edad, al no hallar una representación funcional lógica y teniendo en cuenta la importancia teórica de la distinción de las adolescentes con respecto al resto de las mujeres, se decidió trabajarla como dictómica.

Finalizado el proceso de selección, el modelo elegido es el que contiene los efectos principales de las variables transfusiones de sangre, años de residencia en áreas endémicas, edad y nivel de instrucción. La prueba de Hosmer-Lemeshow proporcionó un valor observado de 9,285 con probabilidad asociada igual a 0,32 .

Las estimaciones de las ROs, de acuerdo al modelo elegido, dan lugar a la tabla 3.

Conforme a lo esperado, los antecedentes vinculados a la infección incrementan notablemente los "odds" para las mujeres infectadas. Así el "odds" de contar con el antecedente de transfusiones de sangre para las mujeres infectadas se incrementa 3 veces en relación al correspondiente a las mujeres no infectadas.

En relación al tiempo de residencia en zonas endémicas, puede apreciarse un incremento en el riesgo a medida que los tiempos son mayores. Así, las ROs son 2,4 para las personas que estuvieron 5 años y 5,6 para las que estuvieron 15 años, en relación con las que no vivieron en zonas endémicas.

Cabe aclarar que se construyó también un modelo de regresión logística en donde se reemplazó años de residencia en áreas endémicas por el antecedente de haber vivido en

Razones de “odds" estimadas de acuerdo al modelo de regresión logística elegido.

\begin{tabular}{lll}
\hline & $\begin{array}{c}\text { Razón de “odds" } \\
\text { (intervalo de confianza del 95\%) }\end{array}$ & Probabilidad asociada \\
\hline Transfusiones de sangre & 1,0 & 0,0026 \\
no & $3,3(1,52 ; 7,33)$ & $0,0000 / 0,0008$ b \\
si & & - \\
Años de residencia en áreas endémicas a & 2,4 & 0,0184 \\
5 años & 5,6 & - \\
15 años & & \\
Edad & 1,0 & 0,0554 \\
menos de 20 años & $2,2(1,15 ; 4,38)$ & 0,0002 \\
20 años o más & & $2,0(0,98 ; 4,07)$ \\
Nivel de instrucción & $3,8(1,88 ; 7,53)$ & \\
secundaria incompleta o más & &
\end{tabular}

a Para esta variable continua, se presentan las razones de "odds" para 5 y 15 años de residencia en área endémica to mando tiempo igual 0 como referencia.

b Probabilidades asociadas a las componentes lineal y cuadrática, respectivamente. 
zonas endémicas (con categorías sí y no). En base a este modelo puede decirse que el antecedente de haber vivido en zona endémica conduce a una RO igual a 3,6.

La RO entre edad de la mujer y condición de infección resultó igual a 2,2, siendo mayor la chance de encontrar mujeres de mayor edad en el grupo de casos. Se confirma, entonces, la relación verificada en el análisis bivariado.

En cuanto a las condiciones socioeconómicas, merece destacarse la importancia del nivel de instrucción de la mujer. Para las mujeres infectadas, se aprecia un incremento en los "odds" (versus las no infectadas) en la medida que el nivel de escolaridad es más bajo. De esta manera, las ROs son 3,8 para las mujeres que no asistieron a la escuela o no completaron la primaria y 2,0 para quienes alcanzaron el nivel primario, en comparación con las que al menos iniciaron el ciclo secundario.

Para la comprensión de la interrelación existente entre algunas variables explicativas, se construyeron una serie de tablas de contingencia, cada una de ellas vinculando el nivel de instrucción con la residencia en villas de emergencia, otra característica socioeconómica y la condición de infección. Se buscó, luego, el modelo loglineal que describiera más adecuadamente la estructura de cada tabla.

A través de los modelos loglineales seleccionados, se llega a la conclusión que la asociación entre la residencia en villas de emergencia y la condición de infección (verificada en forma bivariada) queda totalmente explicada por el nivel de instrucción. En todo segmento de análisis está presente la asociación entre la residencia en villa e instrucción, perdiendo su significación la asociación entre la primera e infección.

Análogamente, se siguió un procedimiento de construcción de tablas similar, en relación a la disponibilidad de agua. La asociación marginal verificada con condición de infección queda explicada por su interacción conjunta con el nivel de instrucción y la residencia en villa.

\section{Conclusiones}

Este estudio corrobora, para la población de mujeres embarazadas del Hospital Roque Sáenz Peña de la ciudad de Rosario, la importancia del antecedente de residencia en áreas endémicas, como el de haber recibido transfusiones de sangre, y se destaca como condicionante socioeconómico preponderante vinculado con la infección el nivel de instrucción de la mujer.

Ante la necesidad planteada por algunos autores (Andrade et al., 1994) de desarrollar estrategias preventivas, identificar grupos de riesgo y conformar diferencias regionales, estos resultados pueden constituir un aporte en relación a un grupo de riesgo específico : las embarazadas, y un área determinada : urbana, sin presencia del vector pero receptora de migrantes provenientes de zonas endémicas.

En cuanto a los aspectos metodológicos, una contribución sustancial de la modelización estadística empleada reside en que permite un análisis simultáneo de variables teniendo en cuenta el aporte relativo de cada una de ellas.

En particular, la modelización logística resultó adecuada tanto por la posibilidad de valorar simultáneamente covariables categóricas y continuas bajo un diseño específico, el de casos y controles, cuanto por proporcionar interpretación epidemiológica a sus coeficientes dada su aproximación al riesgo relativo. Para ahondar en las asociaciones entre ciertas variables explicativas fue satisfactoria la aplicación de modelos loglineales, en tanto permitió comprender por qué al gunos factores no estaban relacionados con la variable respuesta sino a través de otra u otras variables explicativas.

De este modo el recurso analítico empleado permitió no sólo hallar las asociaciones más relevantes entre los factores considerados y la enfermedad de Chagas, sino también lograr una mejor comprensión acerca de la trama de relaciones existentes entre ellos. 


\section{Agradecimiento}

Esta presentación se realizó a partir del proyecto “Implicancias de los condicionantes socioeconómicos, culturales y psicosociales en la enfermedad de Chagas connatal", desarrollado en el Centro de Estudios Sanitarios y Sociales/Asociación Médica de Rosario, con apoyo financiero de PNUD/Banco Mundial/OMS.

Deseo expresar mi agradecimiento a los integrantes del equipo a cargo de la investigación men- cionada, en particular a la Dra. María del Carmen Troncoso y a la Estadística Irene Luppi por el estímulo y la orientación científica recibida. También deseo expresar un sincero reconocimiento a la Estadística Leticia Hachuel, Profesora Titular de la cátedra "Análisis Multivariado Discreto" en la Universidad Nacional de Rosario, por su valiosa participación en mi formación académica.

\section{Referencias}

AGRESTI, A., 1990. Categorical Data Analysis. New York: John Wiley \& Sons.

ANDRADE, A. L.S. S.; ZICKER, F. \& MARTELLI, C. M . T., 1994. An Epidemiological Approach to Study Congenital Chagas' Disease. Cadernos de Saúde Pública, 10 (supl. 2):345-351.

ANDRADE, A. L.S. S.; ZICKER, F.; SILVA, I. G.; SOUZA, J. M. \& MARTELLI, C. M., 1995. Risk factors for Trypanosoma cruzi infection among children in Central Brazil : a case-control study in vector control settings. American Journal of Tropical Medicineand Hygiene, 52(2):183-187.

AZOGUE, E.; LA FUENTE, C. \& DARRAS, C. H., 1985. Congenital Chagas disease in Bolivia: epidemiological aspects and pathological findings. Transactions of the Royal Society of Tropical Medicine and Hygiene, 79:176-180.

BAROUSSE, A. P.; EPOSTO, M. O.; MANDEL, S. \& SOUZA MARTINEZ, F., 1978. Enfermedad de Chagas congénita en área no endémica. Medicina, 38: 611-615.

BITTENCOURT, A., 1976. Congenital Chagas disease. American Journal of Diseases of Childhood, 130: 97-103.

CAMPOS, N. \& FRANCO, E. L., 1989. A microcomputer program for multiple logistic regression by unconditional and conditional maximum likelihood methods (MULTR) User's Manual. American Journal of Epidemiology. 129:439-444.

FAREWELL, V. T., 1979. Some results on the estimation of logistic models based on retrospective data. Biometrika, 66:27-32.

FIENBERG, S., 1981. The Analysis of Cross-classified Categorical Data. Second Edition. Cambridge: Mass, The MIT Press.

HOSMER, D. W. \& LEMESHOW, S., 1989. Applied Logistic Regression. New York : John Wiley \& Sons.

LORCA, M.; BEROIZA, A. M.; MUÑOZ, P.; GUAJARDO, U.; SILVA, J.; CANALES, M. \& ATIAS, A., 1987. Estudio materno infantil de enfermedad de Chagas en zonas endémicas. III: Salamanca, Valle de Choafa, Chile. Parasitología al Día. 11:97-100.

MARTELLI, C. M. T.; ANDRADE, A. L. S. S.; SILVA, S. A. \& ZICKER, F., 1992. Risk factors for Trypanosoma cruzi infection among blood donors in Central Brazil. Memórias do Instituto Oswaldo Cruz, 87: 339-343.
MOYA, P.; PAOLASSO, R. D.; BLANCO, S.; LAPASSET, M.; SANM ARTINO, C.; BASSO, B.; MORETTI, E. \& CURA, D., 1985. Tratamiento de la enfermedad de Chagas con nifurtimox durante los primeros meses de vida. Medicina, 45:553-558.

NORUSIS, M. J., 1986. SPSS/PC+for the IBM PC/XT/ AT. Chicago: SPSS inc.

OLIVIER, D. C. \& NEFF, R. K., 1976. LOGLIN 1.0. Users's guide. Health Sciencies Computer Facility. Boston: Harvard School of Public Health.

SALEME, A. E.; YANICELLI，G. L.; IÑIGO, L. A.; VALPERGA, S. M.; ALONSO, E.; PAZ, A.; MORENO, A.; ZERDAN, A. C.; HATEM , J. \& GARCIA TORO, A., 1971. Enfermedad de Chagas Mazza congénita en Tucumán. Archivo Argentino de Pediatría. 69:162-169.

STARR, M. D.; ROJAS, J. C.; ZELEDON, R.; HIRD, D. W. \& CARPENTER, T. E., 1991. Chagas' Disease: risk factors for house infestation by Triatoma dimidiata, the major vector of Trypanosoma cruzi in Costa Rica. American Journal of Epidemiology, 133:740-747.

TORRES DE QUINTEROS, Z.; TRONCOSO, M. C.; ARNESI, N.; BOGGIO, G. \& SÁNCHEZ, S. M., 1990. Comportamientos migratorios en donantes de sangre y su relación con infección chagásica. Cuadernos Médico Sociales, Rosário, 54:3-14.

TORRES DE QUINTEROS, Z.; TRONCOSO, M. C.; BLOCH, C.; SÁNCHEZ, S. M.; GODOY, C.; APARICIO, V. \& QUINTEROS, A., 1992. Infección chagásica y antecedentes migratorios en la población de villas de emergencia de Rosario. Cuadernos Médico Sociales, 62:1-16.

TRONCOSO, M. C.; BOGGIO, G.; LUPPI, I.; MENEGHINI, M. B.; SÁNCHEZ, S. M. \& SCHAPIRA, M., 1995. Implicancias de los Condicionantes Socioeconómi cos, Culturales y Psicosociales en la Enfermedad deChagas Connatal. Informe final presentado a OMS, Special Programme for Research and Training in Tropical Diseases. Rosario: Centro de Estudios Sanitarios y Sociales.

ZAIDENBERG, M. \& SEGOVIA, A., 1993. Enfermedad de Chagas congénita en la ciudad de Salta, Argentina. Revista do Instituto de Medicina Tropical deSão Paulo, 35:35-43. 